\title{
Contribution of ankle, knee, and hip joints to the perception threshold for support surface rotation
}

\author{
NORMAND TEASDALE \\ Université Laval, Saint Foy, Quebec, Canada \\ VINCENT NOUGIER \\ Université Joseph Fourier, Grenoble, France \\ PIERRE-ALAIN BARRAUD \\ Centre de Recherche du Service de Santé des Armées, La Tronche, France \\ CHRISTOPHE BOURDIN and BETTINA DEBU \\ Université Joseph Fourier, Grenoble, France \\ and \\ DIDIER POQUIN and CHRISTIAN RAPHEL \\ Centre de Recherche du Service de Santé des Armées, La Tronche, France
}

\begin{abstract}
The purpose of the present experiment was to investigate the extent to which subjects can perceive, at very slow velocities, an angular rotation of the support surface about the medio-lateral axis of the ankle, knee, hip, or neck joint when visual cues are not available. Subjects were passively displaced on a slowly rotating platform at $.01, .03$, and $.05 \mathrm{deg} / \mathrm{sec}$. The subjects' task was to detect movements of the platform in four different postural conditions allowing body oscillations about the ankle, knee, hip, or neck joint. In Experiment 1, subjects had to detect backward and forward rotation (pitching). In Experiment 2, they had to detect left and right rotations of the platform (rolling). In Experiment 3, subjects had to detect both backward/forward and left/right rotations of the platform, with the body fixed and the head either fixed or free to move. Overall, when the body was free to oscillate about the ankle, knee, or hip joints, a similar threshold for movement perception was observed. This threshold was lower for rolling than for pitching. Interestingly, in these postural conditions, an unconscious compensation in the direction opposite to the platform rotation was observed on most trials. The threshold for movement perception was much higher when the head was the only segment free to oscillate about the neck joint. These results suggest that, in static conditions, the otoliths are poor detectors of the direction of gravity forces. They also suggest that accurate perception of body orientation is improved when proprioceptive information can be dynamically integrated.
\end{abstract}

How we orient ourselves in space is a fundamental problem for the understanding of upright stance regulation. Under normal circumstances, the perceived orientation of the body with respect to gravity is based on the integration of sensory information from the visual, proprioceptive, and vestibular systems. A common assumption is that the center of gravity projection onto the ground is one of the external regulated values available to determine postural stability (see Massion, 1992, for a review). The question of how this regulated value is computed, however, remains a matter of debate. One hypothesis is that otolith organs are graviceptors (Young, 1984) or graviceptive organs (Platt, 1984) that provide information about the direction of gravity forces and the orientation of the body with respect to them. This argument is based on the premise that

Correspondence should be addressed to V. Nougier. Lniversité Joseph Fourier, UFRAPS, BP 53, 38041 Grenoble cedex 9, France (e-mail: vincent.nougier@ujf-grenoble.fr).

--Accepted by previous editor, Myron L. Braunstein the otoliths can directly compute the projection of the center of gravity onto the base of support. Stoffregen and his co-workers (Riccio, Martin, \& Stoffregen, 1992; Stoffregen \& Riccio, 1988) have argued that this cannot be the case because gravitational stimulation of the otolith organs gives rise only to a poor perception of the direction of gravity. For example, when immersed in the water-that is, when the visual and somatosensory systems have limited access to gravity-based information-subjects make errors in body orientation that are greater than $15 \mathrm{deg}$, and within a range of $180 \mathrm{deg}$ (Nelson, 1968; Ross, Crickmar, Sills, \& Owen, 1969). More recently, Fitzpatrick and McCloskey (1994) have determined a vestibular threshold for the perception of sway during quiet standing. Vision was excluded and the whole body was moved with the ankles in a fixed position. The vestibular threshold was many times greater than the visual or proprioceptive thresholds. Furthermore, the vestibular threshold was above the largest sway movements that were recorded during normal standing. Conversely, visual and proprioceptive thresholds were 
often smaller than sway movements during normal standing. ${ }^{1}$ These results suggested to the authors that the vestibular system plays no part in the perception of sway during normal standing. Hence, there are several arguments against the idea that body orientation is directly computed from gravity signals generated by the vestibular system.

Modular and body schema approaches to the study of body posture have proposed that body orientation arises from the comparison of a body schema, partly genetically determined and partly acquired through learning, to a representation of the verticality based on the continuous integration of visual, labyrinthine, and proprioceptive inputs. Within this general framework, the basic assumption is that a sensorimotor mode of processing spatial information coexists with a representational mode (Paillard, 1987). In these models, the body is not a simple rigid block oscillating like an inverted pendulum about the ankle joint. Rather, it consists of linked superimposed modules from the feet to the head. The linkage between the modules is ensured by muscles that have their own specific central and peripheral regulation, to maintain the modules' reference position (Massion, 1992). More specifically, the representation of body geometry is based on muscle proprioceptive Ia inputs that convey information about the position of a given segment with respect to the others. According to this view, all movements of our articulated body are calibrated within the proprioceptive field of a postural space (Paillard, 1991). The body schema is referred to an internal representation of the extrapersonal space and to the characteristics of the environment (e.g., vision vs. no-vision environment or earth's surface vs. orbital ambiant). Correction of external as well as internal disturbances are based on comparison of these references.

In the present three experiments, we investigated the extent to which subjects can consciously perceive slow angular rotation of the support surface about the mediolateral and antero-posterior axes of the ankle, knee, hip, and neck joints when visual cues are not available. It is known that conscious perception occurs at a higher threshold than subconscious detection. These subconscious detections seem to be the basis of the reflex postural control (Fitzpatrick, Taylor, \& McCloskey, 1992). It is probable, however, that if conscious perception is affected by the manipulation of different experimental conditions, unconscious detection is modified in a similar way when placed in the same conditions. In Experiment 1 (pitching), subjects were displaced in four different postures. In three of them, the body was free to oscillate about the medio-lateral axis of the ankle, knee, or hip joint. In the last condition, subjects were seated and secured on the support surface so as to prevent body oscillations with the exception of the head. Experiment 2 (rolling) replicated Experiment 1, with body oscillations about the antero-posterior axis of the ankle, knee, hip, and neck joints. In Experiment 3, subjects had to detect both backward/forward and left/right rotations of the platform, with the body fixed and the head either fixed or free to move. Overall, it was hypothesized that thresholds for perceiving a movement would be lower when the body was free to oscillate about a given joint than when it was fixed. In this latter postural condition, we hypothesized that vestibular mechanisms were not activated by the motion resulting from postural dynamics, and that proprioceptive information (except for cutaneous information) was severely reduced. It was also hypothesized that the perception of the orientation of the support surface with respect to the body should not be ankle specific, since each module constituting the body schema has its own reference.

\section{GENERAL METHOD}

\section{Apparatus}

Subjects stood on a rigid L-shaped platform (Figure 1). The horizontal panel was $1.5 \mathrm{~m}$ wide and $1.2 \mathrm{~m}$ long. The vertical panel was $1.5 \mathrm{~m}$ wide and $2 \mathrm{~m}$ high. The platform was fixed, $50 \mathrm{~cm}$ above the ground, on computer-motorized axes allowing pitching and rolling movements at various velocities. The axes of rotation were situated in the middle of the vertical panel, $1 \mathrm{~m}$ above the horizontal support surface. Position signals from the platform were sampled at $20 \mathrm{~Hz}$ (12-bit A/D converter). Emergency buttons at different locations of the experimental room enabled subjects to stop the rotation of the platform immediately if needed. A hand-held button also allowed the subjects to stop the platform.

\section{Procedure}

The subjects' task was to detect whether the platform had moved. The detection was recorded by way of pressing the hand-held button that stopped the rotation. Subjects also had to indicate the direction of the platform movement. Three different velocities for the platform rotation were used: $.01, .03$, and $.05 \mathrm{deg} / \mathrm{sec}$. The detection task was executed in different postural conditions allowing body oscillations about a given joint.

Prior to each trial, subjects closed their eyes and stabilized their posture in the required position. When they were ready, the trial was initiated, and it ended with the detection of a platform rotation. It has been emphasized elsewhere (e.g., Fitzpatrick \& McCloskey, 1994; Hall \& McCloskey, 1983; Melvill-Jones \& Young, 1978) that movement perception can occur before the direction of the movement can be reported. The direction of the movement, however, also has to be perceived if this detection is to be functionally useful. Furthermore, it is likely that processing at a subconscious level occurs before conscious perception. These subconscious detections have been proposed to form the basis of reflex postural control (Fitzpatrick et al., 1992). For these reasons, prior to opening their eyes at the end of each trial, subjects indicated the direction in which the platform had moved as well as their level of confidence on a 5-point scale. Subjects were encouraged to stop the rotation of the platform (i.e., to press the hand-held button) when their level of confidence exceeded that of simple chance. Following the response, the platform was brought back to the starting position.

\section{EXPERIMENT 1}

In this first experiment, we investigated the threshold for movement perception for backward and forward rotations (pitching) when the body was free to oscillate about the ankle, knee, hip, or neck joint. It was hypothesized that head oscillations about the neck joint would induce greater thresholds for movement perception than body oscilla- 


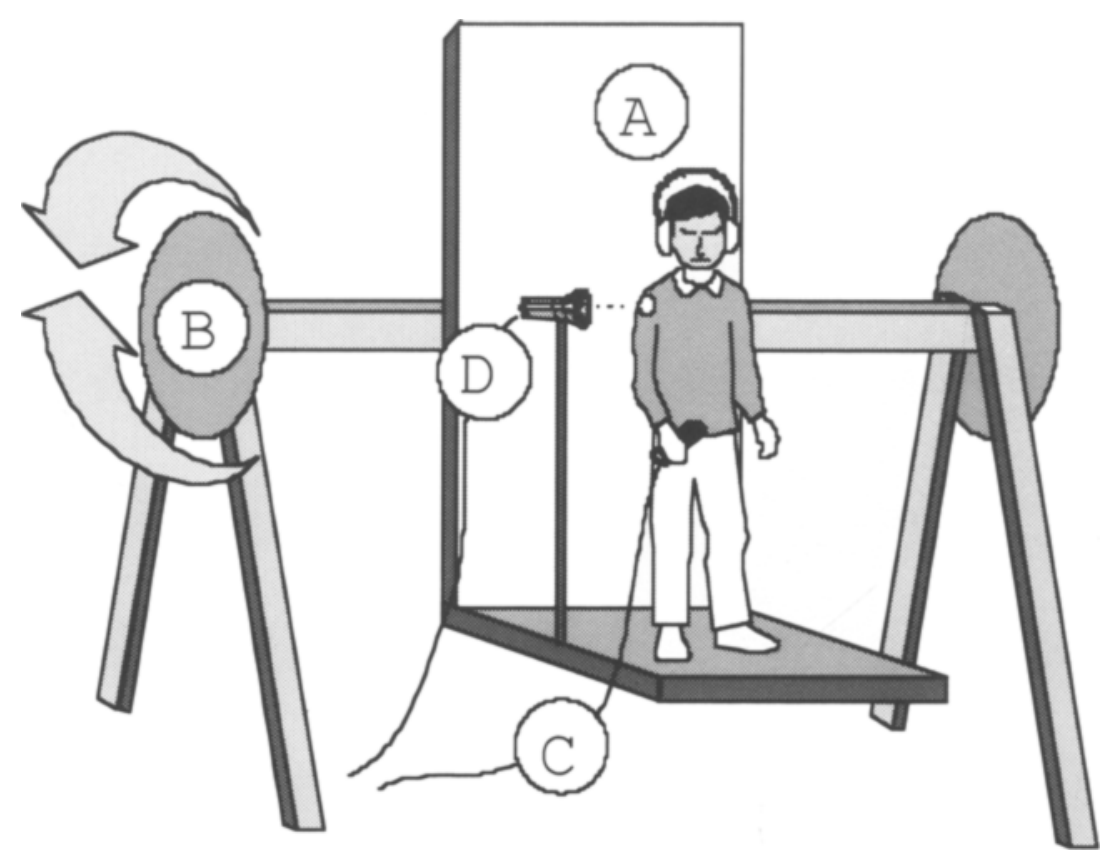

Figure 1. The apparatus was an L-shaped platform (A), rotating about an axis via a computer-controlled servomotor (B). A hand-held button (C) allowed the subject to stop the platform. In addition, a Hamamatsu infrared camera (D) was fixed laterally on the frame of the platform, $80 \mathrm{~cm}$ from the subject. This camera was used to record body oscillations, via an infrared light emitting diode (IRED) located on the shoulder.

tions about the ankle, knee, or hip joint, because of the absence of global body oscillations in the neck condition.

\section{Method}

Subjects. Four subjects $(3$ males and 1 female, mean age $=$ 33 years) participated in the experiment. All subjects gave informed consent. All were naive about the hypotheses being tested and about the nature of the research in general.

Procedure. The detection task was executed in four postural conditions allowing body oscillations about the ankle, knee, hip, or neck joint with eyes closed and earphones providing a white noise. In the ankle condition, subjects stood barefoot $40 \mathrm{~cm}$ in front of the vertical panel of the platform, feet together. In this condition, the subject's body was free to oscillate about the medio-lateral axis of the ankle joint. In the knee condition, subjects stood kneeling, the chest and the thigh being aligned vertically. In this condition, the upper part of the body could oscillate freely about the medio-lateral axis of the knee joint, the contribution of ankle joint information to the control of posture being excluded. In the hip condition, subjects sat on a stool. The subject's chest was free to oscillate about the medio-lateral axis of the hip joint. In this condition, the contribution of ankle and knee joint information to the control of posture was excluded. In the neck condition, subjects were seated against the back of the platform and were attached to it with shoulder and hip straps. Body pressure was largely distributed between (1) the back against the platform, (2) the shoulders and hips against the straps, and (3) the buttocks and thighs on the seat. In this condition, the platform could rotate without any oscillation of the body. Only the head was free to oscillate about the medio-lateral axis of the neck joint. This condition allowed comparison of the subject's threshold for movement perception when the body was almost completely fixed and when it was free to oscillate about a given joint.
The four postural conditions were tested in four separate blocks of trials. For each postural condition, three trials for each direction and each velocity were executed randomly. Two catch trials with the platform remaining immobile were also inserted randomly in each block, for a total of 20 trials. These catch trials were used to ensure that subjects did not detect movements of the platform by guessing. The blocks of trials were presented randomly on 2 separate days. Each experimental session lasted approximately $2 \mathrm{~h}$. Resting periods were provided between trials, when the platform was brought back to the horizontal 0 deg position, and whenever necessary.

In addition, a Hamamatsu infrared camera was fixed laterally on the frame of the platform, $80 \mathrm{~cm}$ away from the subject. Thus, the camera moved together with the platform. This camera was used to record body oscillations, via an infrared light emitting diode (IRED) located on the shoulder, at the extremity of the acromion. Body oscillations were sampled at $20 \mathrm{~Hz}$ (12-bit $\mathrm{A} / \mathrm{D}$ converter).

\section{Results and Discussion}

On the average, subjects made detection errors on $14 \%$ of all trials. Subjects detected a movement of the platform but gave the wrong direction of rotation for $27 \%$ of these errors. The remaining $73 \%$ of the errors occurred when catch trials were given, meaning that subjects detected a movement of the platform, although it did not move. For these trials, subjects reported a forward rotation of the platform in most cases ( $80 \%$ of the errors on catch trials). This means that body oscillations were sometimes interpreted as resulting from a rotation of the platform.

A 3 (velocities: $.01, .03$, and $.05 \mathrm{deg} / \mathrm{sec}$ ) $\times 4$ (postures: ankle, knee, hip, and neck) $\times 2$ (directions: forward 


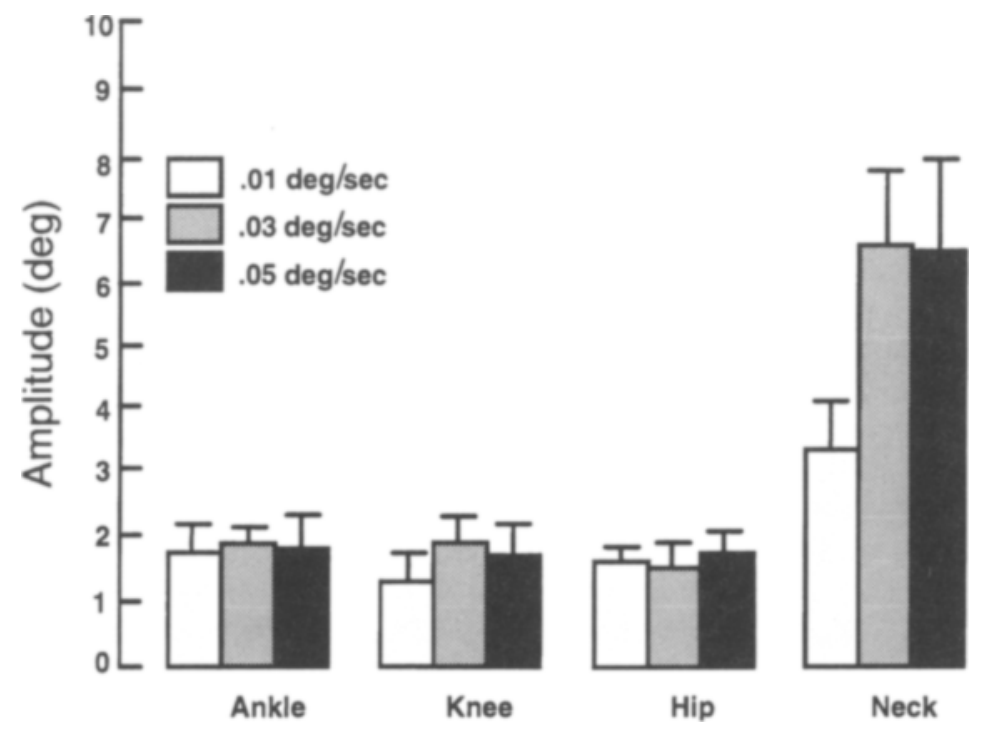

Figure 2. Means and standard deviations for the angular positions at which subjects perceived a rotation of the platform when oscillating about the mediolateral axis of the ankle, knee, hip, or neck joint (pitching) at the three different velocities.

and backward) analysis of variance (ANOVA) with repeated measures on all factors was applied to the detected positions. Results showed main effects of velocity $[F(2,6)=6.23, p<.05]$ and posture $[F(3,9)=19.77, p<$ $.001]$, and a significant velocity $\times$ posture interaction $[F(6,18)=5.25, p<.01]$. The main effect of direction and its interaction with other factors were nonsignificant $(p s>.05)$. As illustrated in Figure 2, there was no difference in thresholds for movement perception for the three conditions in which the subject's body could oscillate freely about a given joint $(1.73,1.63$, and $1.59 \mathrm{deg}$, for the ankle, knee, and hip conditions, respectively). Similar results were observed for the three velocities. Conversely, the neck condition yielded much higher threshold values ( $5 \mathrm{deg}$ ). In this condition, the threshold values were affected by velocity. At the two fastest velocities (.03 and $.05 \mathrm{deg} / \mathrm{sec}$ ), thresholds for movement perception were significantly higher in the neck condition than in the other three conditions $(p s<.001)$. At the slowest velocity $(.01 \mathrm{deg} / \mathrm{sec})$, threshold for movement perception was twice that observed when body oscillations were present. However, comparisons of means showed that this difference was not significant ( $p$ s $>.05$ ).

These results showed that when body oscillations were absent or very limited, threshold for movement perception increased considerably. Furthermore, they showed that when the body was free to move about the ankle, knee, and hip joints, threshold for movement perception was not different. In all postures, however, thresholds were more than 10 times higher than those reported in similar experiments. More specifically Blaszczyk, Hansen, and Lowe (1993) reported matching errors between one foot and the other of $0.1 \mathrm{deg}$ on the average, and Fitzpatrick and McCloskey (1994) reported thresholds for move- ment perception of $0.06-0.12 \mathrm{deg}$. On the other hand, it has been shown that subjects immersed in a swimming pool make errors in the perception of the gravitational vertical that are much greater than those reported in the present experiment (e.g., Nelson, 1968; Ross et al., 1969).

Our results clearly indicate that otolith sensitivity to gravity-based information, if useful, is not sufficient for a good perception of the orientation of the body in space. This finding also supports Fitzpatrick and McCloskey's (1994) claim that vestibular inputs play no part in the perception of normal body sway. If one assumes that in all experimental conditions otolith organs were stimulated similarly by the rotation of the platform, the thresholds for movement perception should have been independent of the posture. In the seating-fixed condition, however, the subject's threshold for perception of movement was higher than when the subject's body was free to oscillate about a joint. When subjects were attached, only otolithic information (if available), small oscillations about the neck joint, and variations of pressure on different parts of the body were available. Our results show that, in a very static posture, the otoliths cannot correctly inform the subjects about the direction of gravity forces with respect to the body-that is, they cannot be viewed as precise graviceptors (Young, 1984) or graviceptive organs (Platt, 1984). These results also demonstrate that gravity-based information, at least for slow velocities of rotation, has to be dynamically integrated. One cannot exclude that vestibular signals contributed to the perception of the platform rotation when subjects were free to oscillate. The direction of gravity forces, however, seems to be more precisely integrated via proprioceptive information from active agonist and antagonist muscles. 
Some have suggested that the direction of upright stance is mainly dependent on an unstable equilibrium (Riccio et al., 1992; Stoffregen \& Riccio, 1988). That is to say, there does not exist an objective vertical, and body instability is a necessary source of information to achieve a good orientation of the body in space. Results of the present experiment partly support this view since thresholds for movement perception were lower when the body was free to oscillate than when it was fixed, independently of the main joint about which the body could oscillate. This observation suggests that body oscillations constitute a significant signal provided to the system for perceiving movement and for orienting the body in space. An almost constant signal-to-noise ratio may explain that thresholds for movement perception were similar for the different joints and muscles involved in body oscillations.

Another point regarded subjects' behavior when the platform was moving. Interestingly, when the body was free to oscillate, an unconscious compensation in the direction opposite to that of the platform rotation was observed on most trials. Representative trials for platform rotation and body oscillations in the ankle, knee, and hip conditions are presented in Figure 3. The good negative correlation between rotation of the platform and body compensation is clear.

Again, the signal-to-noise ratio from the muscle spindles involved in the control of posture may explain how the compensatory mechanism occurs. The rotation of the platform did, indeed, perturb subjects' equilibrium. This probably modified the length-tension relationship for the agonist and antagonist muscles participating in the regulation of posture. It is possible that the signal-to-noise ratio originating from this length-tension relationship activated the compensatory mechanism in order to keep the signal-to-noise ratio rather constant or close to its reference value. As suggested by several authors (Clément, Gurfinkel, Lestienne, Lipshits, \& Popov, 1984; Lestienne \& Gurfinkel, 1988; Massion, 1992; Paillard, 1990), with experience, subjects elaborate an internal reference based in part on information originating from gravity forces. It is possible that subjects integrate such a reference, not (or not only) by means of specific receptors such as the otoliths, but also by means of the dynamic signals originating from the muscles activated during body oscillations. These dynamic signals would not inform the subjects about the absolute or Euclidean vertical, but rather would define the stability margins beyond which a fall may occur. Therefore, the subjects' internal reference could be viewed as a sort of vertical cylinder within which body oscillations can occur without risk of threatening balance (Collins \& De Luca, 1993). When the signal-to-noise ratio differs from this reference value, unconscious compensatory adjustments occur to adapt to the environmental constraints and to prevent a fall. When this signal-to-noise ratio exceeds a given value, movement perception may occur.

Fitzpatrick and McCloskey's (1994) results have shown that thresholds for the perception of movement are dependent on the velocity of the imposed movement of the body (from .057 up to $.17 \mathrm{deg} / \mathrm{sec}$ ). In their experiment, thresholds decreased as movement velocity increased, suggesting that subjects perceived movement rather than a new position. In the present experiment, no effect of velocity was observed, at least when the subject's body was free to oscillate. At least two explanations can account for this discrepancy. On the one hand, we tested slower velocities than did Fitzpatrick and McCloskey. This may suggest a velocity threshold below which the system is not sensitive enough to movement. On the other hand, in our experiment the movement was applied to the platform and indirectly affected body orientation and oscillations, whereas in Fitzpatrick and McCloskey's experiment, the movement was imposed on the body, directly perturbing body oscillations. This difference in experimental protocol may have induced a drift in the threshold for movement perception or a difference in the oscillation dynamics.

However, the significant velocity $\times$ posture interaction showed, in the seating-fixed condition, a lower threshold for movement perception at the slowest velocity $(.01 \mathrm{deg} /$ sec). In this condition, cutaneous receptors provided the most significant sensory information, since the pressure of the body against the platform or the belts increased with the platform rotation. It is known that cutaneous receptors mediating the sense of touch can be divided into two functional groups (see Martin \& Jessell, 1991, for a review). The so-called rapidly adapting receptors respond to the onset and offset of the stimulating pressure. The slowly adapting receptors, particularly stimulated in the present experimental conditions, respond continuously to a maintained pressure. With respect to muscles la afferent fibers, cutaneous conduction velocity is more than two times slower. This may explain the significant effect of velocity observed in the seating-fixed condition. At the slowest velocity, cutaneous receptors had time enough to provide the central nervous system with a signal that could be used to perceive a movement of the platform. At the other two velocities, the response delay of the cutaneous pathway was too long, with respect to the rotation of the platform, to provide a signal that could be integrated and used immediately.

\section{EXPERIMENT 2}

The purpose of Experiment 2 was to validate the results of Experiment 1 for rolling instead of pitching movements. Since body oscillations are more restricted about the antero-posterior than about the medio-lateral axis of joint rotation (Paulus, Straube, \& Brandt, 1984), we expected lower thresholds for movement perception in rolling than in pitching, when the body was free to oscillate about the ankle, knee, or hip joint. On the other hand, a similar threshold was also hypothesized for movement perception in rolling and pitching when the body was free to oscillate about the neck joint, because of similar amplitudes of head movements about the anteroposterior and medio-lateral axes of neck rotation. 

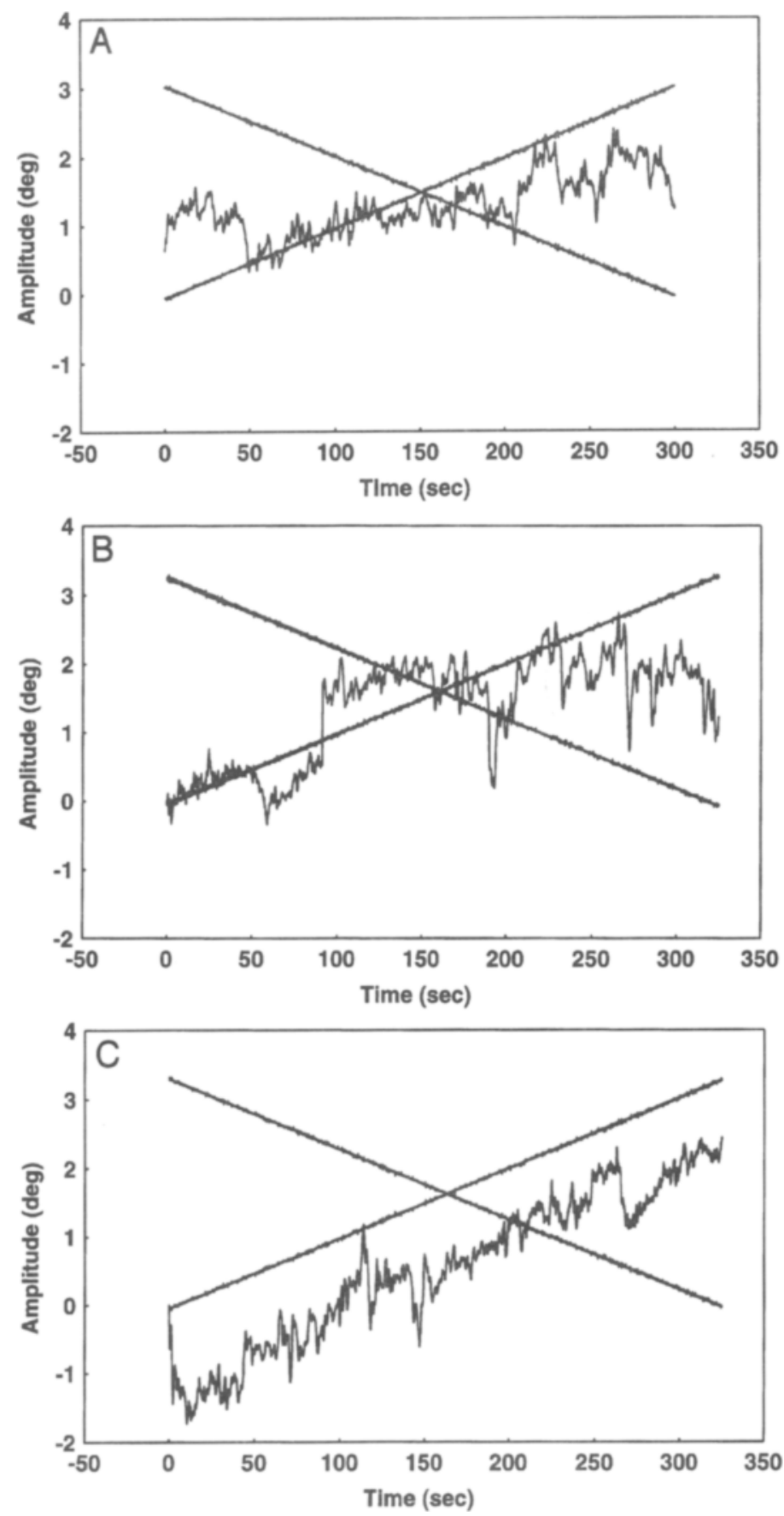

Figure 3. Representative trials for the ankle (A), knee (B), and hip (C) conditions at the $.01 \mathrm{deg} / \mathrm{sec}$ velocity. The decreasing slope represents the linear rotation of the platform within time when the platform was inducing a forward inclination. There was a good negative correlation between platform rotation and spontaneous body compensation (for illustration purposes, movement of the platform was also reported symmetricallyincreasing slope-and superimposed with body oscillations). Overall, when the platform was rotating forward, the subject's center of gravity was rotating backward with respect to movement of the platform. More specifically, when body oscillations went in the direction opposite to platform rotation (toward the upper part of the ordinate axis), the subject was compensating for the platform rotation. Conversely, when body oscillations went in the same direction as the platform rotation (toward the lower part of the ordinate axis), the subject's body was globally following the movement of the platform. 


\section{Method}

Subjects. Five new subjects ( 3 males and 2 females, mean age $=$ 30 years) participated in Experiment 2. All subjects gave informed consent and were naive about the purpose of the experiment.

Procedure. The apparatus and task were the same as in Experiment 1. Body oscillations, however, were not recorded. Movements of the platform induced either left or right rotations (rolling) at the same three velocities $(.01, .03$, and $.05 \mathrm{deg} / \mathrm{sec})$. The detection task was executed in the same four postural conditions, allowing body oscillations about the ankle, knee, hip, or neck joint, with eyes closed and earphones providing a white noise. The four postural conditions were tested in four separate blocks of trials. For each postural condition, three trials for each direction and each velocity were administered randomly. Two catch trials with the platform remaining immobile were also included in each block, for a total of 20 trials. The blocks of trials were presented randomly on 2 separate days. Each experimental session lasted approximately $2 \mathrm{~h}$. The platform was brought back to the vertical 0 deg position during resting periods, which were provided between trials and whenever necessary.

\section{Results and Discussion}

On the average, subjects made detection errors-that is, detected a movement of the platform but gave the wrong direction of rotation-on $7 \%$ of the trials.

A 3 (velocities: $.01, .03$, and $.05 \mathrm{deg} / \mathrm{sec}$ ) $\times 4$ (postures: ankle, knee, hip, and neck) $\times 2$ (directions: left and right) ANOVA with repeated measures on all factors was applied to the detected positions. Results showed main effects of velocity $[F(2,8)=28.65, p<.001]$ and posture $[F(3,12)=46, p<.001]$, and a significant velocity $\times$ posture interaction $[F(6,24)=9, p<.001]$. The main effect of direction and its interaction with other factors were nonsignificant ( $p \mathrm{~s}>.05$ ). As illustrated in Figure 4, there was no difference in thresholds for movement perception for the three conditions in which the subject's body could oscillate freely about a given joint $(.6, .95$, and $.7 \mathrm{deg}$ for the ankle, knee, and hip conditions, respectively). Similar results were observed for the three velocities. Conversely, the neck condition yielded much higher threshold values $(2.95 \mathrm{deg})$. In this condition, threshold for movement perception increased as platform velocity increased $(p s<.01)$.

Overall, the results of Experiment 2 confirmed those of Experiment 1 . When body oscillations were almost absent (neck condition), threshold for movement perception increased considerably. When the body was free to move about the ankle, knee, and hip joints, thresholds for movement perception were not different. These findings confirmed the idea that the direction of upright stance is mainly dependent on an unstable equilibrium (Riccio et al., 1992; Stoffregen \& Riccio, 1988). These results also supported the hypothesis that otolith sensitivity is not sufficient for a good perception of the orientation of the body in space. As claimed by Fitzpatrick and McCloskey (1994), vestibular inputs did not seem to contribute significantly to the perception of normal body sway.

Interestingly, threshold for movement perception was approximately twice as low in rolling than in pitching. This was true for all joint conditions. Two hypotheses, at least, can account for these results. According to the first hypothesis, subjects are more used to modifying the orientation of the body in space about the medio-lateral than about the antero-posterior axis of joint rotation. As a result, the level of tolerance - that is, the subjects' capacity to adapt to new constraints and therefore the threshold for movement perception - is higher in pitching than in rolling, confirming Riccio et al.'s (1992) hypothesis.

According to the second hypothesis, the lower the possible amplitude of body oscillations, the lower the threshold for movement perception, in order to prevent the subject from falling. This may explain the lower thresholds observed for body oscillations about the antero-posterior rather than about the medio-lateral axes of the ankle, knee, and hip joints. When body oscillations are smaller (Paulus et al., 1984) as in rolling, body compensation in the direction opposite to that of the platform rotation is limited, too, because of the more restricted joint amplitude. As noted, the signal-to-noise ratio originating from the muscle spindles involved in the control of posture may explain how the compensatory mechanism works. When the signal-to-noise ratio differs from a reference value, compensatory adjustments occur until this ratio exceeds a given value. Then, movement perception may occur. It is possible that the critical value that prevents falls is reached earlier in rolling than in pitching.

\section{EXPERIMENT 3}

The results of Experiments 1 and 2 showed that when body oscillations are absent or at least very limited (neck condition), threshold for movement perception increases considerably. The purpose of Experiment 3 was to further investigate the contribution of the head to the detection of platform rotations. Threshold for movement perception was measured in two seated conditions, with the head fixed and the head free to move. It was hypothesized that threshold for movement perception would be higher when the head was fixed, in absence of any movement of the body.

\section{Method}

Subjects. Four new subjects ( 3 males and 1 female, mean age $=$ 32 years) participated in the experiment. All subjects gave informed consent and were naive as to the purpose of the experiment.

Procedure. The apparatus and task were the same as in Experiments 1 and 2. Body oscillations were not recorded. Movements of the platform induced either left/right rotations (rolling) or backward/ forward rotations (pitching) at a velocity of $.05 \mathrm{deg} / \mathrm{sec}$ only. This velocity was chosen because it was the one inducing the highest thresholds for movement perception in the neck condition. The detection task was executed in two postural conditions. The head-free condition replicated the neck condition of Experiments 1 and 2. The head was free to move about the antero-posterior and medio-lateral axes of the neck joint. In the head-fixed condition, subjects wore a helmet fixed to the platform to prevent any head movement about any axis of the neck joint. In that condition, subjects were completely immobilized. For each block of trials (four blocks in all), four trials in each direction of rolling or pitching were executed randomly. Two catch trials with the platform remaining immobile were 


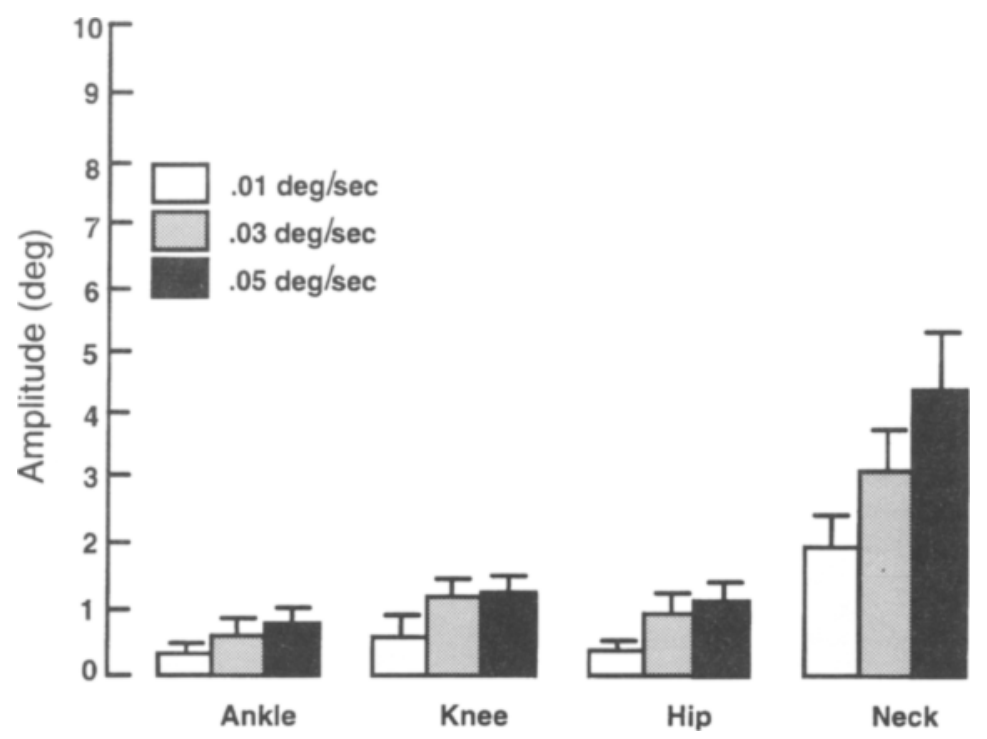

Figure 4. Means and standard deviations for the angular positions at which subjects perceived a rotation of the platform when oscillating about the anteroposterior axis of the ankle, knee, hip, or neck joint (rolling) at the three different velocities.

also inserted in each block for a total of 10 trials. The four blocks of trials were presented randomly on 2 separate days. Each experimental session lasted about $45 \mathrm{~min}$. The platform was brought back to the vertical $0 \mathrm{deg}$ position during resting periods, which were provided between trials and whenever necessary.

\section{Results and Discussion}

On the average, subjects made detection errors on $8 \%$ of the trials-that is, detected a movement of the platform but gave the wrong direction of rotation. A 2 (postures: head free and head fixed) $\times 2$ (directions: pitching and rolling) ANOVA with repeated measures on both factors was applied to the detected positions. The main effects of posture $[F(1,3)=3.7, p>.10]$ and direction $[F(1,3)=$ $6.47, p>.10]$ and their interaction $[F(1,3)=0.19, p>$ $.10]$ were nonsignificant. As illustrated in Figure 5, there was no difference in thresholds for movement perception for the two head conditions and for the two directions of platform rotation.

Overall, these results suggest that head oscillations do not contribute significantly to the detection of the threshold for movement perception. It is probable that head oscillations do not usually contribute to the regulation of postural stability. It is well known that head position in space is very important for orienting the whole body in space (Paillard, 1991; Pozzo, Berthoz, \& Lefort, 1990). Our results, however, show that in the absence of vision, the contribution of the head to the orientation of the body in space is not sufficient; body oscillations are also required. The present findings suggest that dynamic signals from the body (Riccio et al., 1992) are necessary to integrate the position signals issued from the orientation of the head in space in order to allow a good orientation of the whole body in space (Pozzo et al., 1990). In other words, the signals issued from the different sensory systems may be partially redundant for orienting the body in space. It remains, however, that they are also complementary, assuming that the integration of these sensory signals has to be dynamically realized.

\section{GENERAL DISCUSSION}

The purpose of the present experiments was to investigate the thresholds at which subjects can perceive rotations of the support surface away from the vertical defined by the forces of gravity. When the body was free to oscillate about a given joint (ankle, knee, or hip), thresholds for movement perception were lower than when subjects were in a fixed posture. This was true both for rolling and for pitching. In all postures, however, thresholds were more than 10 times higher than those reported in other experiments (Blaszczyk et al., 1993; Fitzpatrick \& McCloskey, 1994). However, it has also been shown that subjects immersed in the water (Nelson, 1968; Ross et al., 1969) made errors in the perception of the gravitational vertical that were much greater than those observed in the present experiments.

Otolith sensitivity to gravity-based information did not seem to afford a good perception of the body orientation in space, supporting the idea that vestibular inputs play no part in the perception of normal body sway (Fitzpatrick \& McCloskey, 1994). If one assumes that in all three experiments, otolith organs were stimulated similarly by the rotation of the platform, the thresholds for movement perception should have been independent of the posture. However, in the head conditions, the subject's threshold for movement perception was higher when the body was not free to move than when the body was free to oscillate about a given joint. This suggested that the otoliths can- 


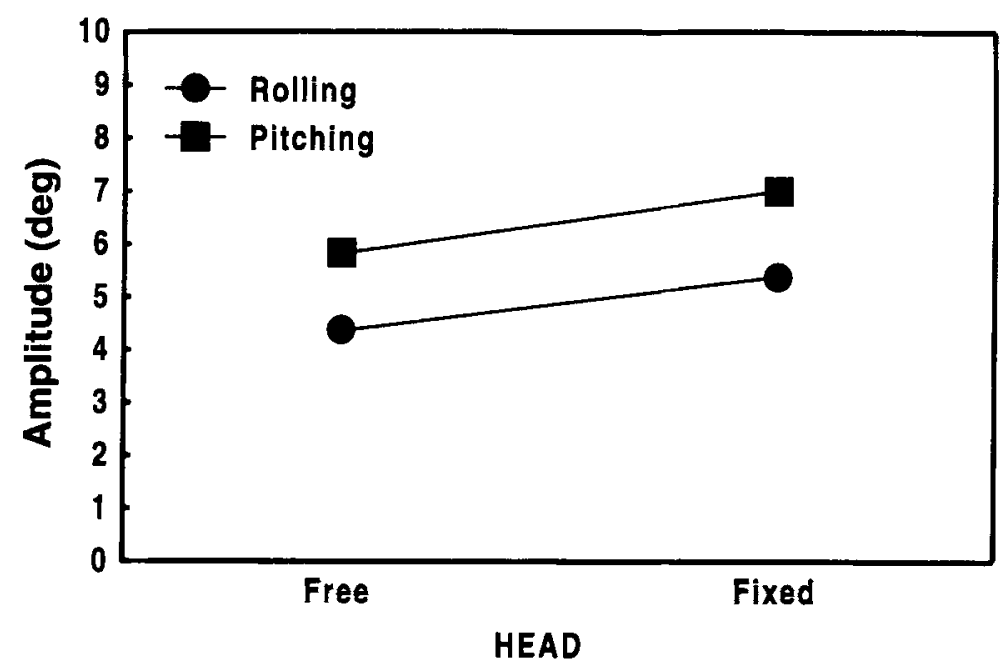

Figure 5. Means and standard deviations for the angular positions at which subjects perceived a rotation of the platform (pitching and rolling) in the head-fixed and head-free conditions.

not be considered as precise graviceptors (Young, 1984) or graviceptive organs (Platt, 1984). This view is consistent with recent findings showing that patients with acute, uncompensated unilateral vestibular lesions did not make greater errors than control subjects in estimating the subjective postural verticality (Anastasopoulos, Haslwanter, Bronstein, Fetter, \& Dichgans, 1997).

These results also demonstrated that gravity-based information, at least for slow velocities of rotation, has to be dynamically integrated via proprioceptive information from active agonist and antagonist muscles. Riccio et al. (1992) have suggested that the direction of upright stance is mainly dependent on an unstable equilibrium. The results of the present experiments partly support this view, since thresholds for movement perception were lower when the body was free to oscillate than when it was fixed, independently of the main joint about which the body could oscillate. Body oscillations seemed to provide a significant signal to the system for perceiving movement and for orienting the body in space. An almost constant signalto-noise ratio may explain that thresholds for movement perception were similar for the different joints and muscles involved in body oscillations.

Paillard (1990) distinguished different frames of reference useful for stabilizing the body. The geocentric frame is based on gravity forces and reaction forces of the supporting surface. The egocentric frame of reference is continuously updated with respect to the position of the different segments. The exocentric frame is based on the external space used as a reference value. A correct body orientation in space depends on the linkage between these different frames of reference. This linkage has to be realized dynamically. Arguments have been provided that body geometry and its dynamics (e.g., Goodwin, McCloskey, \& Matthews, 1972; Hall \& McCloskey, 1983; Roll \& Roll, 1988) are based on signals arising from mus- cle spindles. For example, Roll and Roll proposed that afferent signals from muscle spindles constitute a kinematic chain from the feet to the head and eyes. As shown by various authors, the artificial vibratory stimulation of proprioceptive receptors gives rise to an illusion of movement or to postural adjustments (e.g., Goodwin et al., 1972; Lackner, 1988; Roll \& Roll, 1988). From another point of view, Worringham and his colleagues (Worringham, Stelmach, \& Martin, 1987) have shown that limb segment inclination was perceived much more accurately when matched to the vertical than when matched to joint angles. The authors concluded that muscle length and tension as well as joint reaction forces were probably the most significant signals for perceiving limb orientation in space. However, the appropriate linkage between the different modules constituting the body, or between the different frames of reference, cannot always be correctly updated. For example, the high percentage of errors of movement perception observed in the case of catch trials suggested that body oscillations per se are sometimes interpreted as a rotation of the platform. In other words, the proprioceptive and vestibular systems were not sensitive enough to distinguish between oscillations of the body with respect to the platform and a rotation of the platform with respect to the body.

Fitzpatrick and McCloskey (1994) have shown that when more than one sensory modality is available, subjects perform no better than they would have if only the modality with the greater sensitivity had been available. These authors have proposed that subliminal inputs from two sensory sources are not mutually facilitatory in a way that can increase perceptual experience. When multiple sensory inputs are available, all but the most sensitive one are redundant. This interpretation would support a model in which postural stability is a process resulting from the construction of a body schema or egocentric frame of ref- 
erence and of an exocentric frame of reference (Massion, 1992; Paillard, 1987). The construction of the body schema is multisensorial and, depending on the conditions, one sensory modality dominates over the others.

In conclusion, the most striking result of the present experiments is that, for very slow velocities, threshold for movement perception of the platform was independent of the main articulation about which the body could oscillate. Furthermore, the threshold was lower when the subject's body was free to oscillate than when oscillations were restricted to the head or altogether impossible. This suggested that dynamic signals issued from various receptors (mainly muscular spindle receptors, in this experiment) are necessary for calibrating and updating the subject's motor space with respect to the environment (Bard, Fleury, Teasdale, Paillard, \& Nougier, 1995). It was also shown that, at slow velocities, the vestibular system is a poor detector of gravity forces and thus of the body orientation with respect to the gravity field. Finally, head position signals were useful for body orientation in space only if these signals were integrated with other sensory signals through dynamic processes.

\section{REFERENCES}

Anastasopoulos, D., Haslwanter, T., Bronstein, A., Fetter, M., \& Dichgans, J. (1997). Dissociation between the perception of body verticality and the visual vertical in acute peripheral vestibular disorders in humans. Neuroscience Letters, 233, 151-153.

Bard, C., Fleury, M., Teasdale, N., Paillard, J., \& Nougier, V. (1995). Contribution of proprioception for calibrating and updating the motor space. Canadian Journal of Physiology \& Pharmacology, 73, 246-254.

BlaszczyK, J. W., Hansen, P. D., \& Lowe, D. L. (1993). Accuracy of passive ankle joint positioning during quiet stance in young and elderly subjects. Gait \& Posture, 1, 211-215.

Clément, G., Gurfinkel, V. S., Lestienne, F., Lipshits, M. I., \& Popov, K. E. (1984). Adaptation of postural control to weightlessness. Experimental Brain Research, 57, 61-72.

Collins, J. J., \& DE LuCA, C. J. (1993). Open-loop and closed-loop control of posture: A random-walk analysis of center-of-pressure trajectories. Experimental Brain Research, 95, 308-318.

FitzPatrick, R., \& McCloskey, D. I. (1994). Proprioceptive, visual and vestibular thresholds for the perception of sway during standing in humans. Journal of Physiology, 478, 173-186.

FitzPatrick, R., TAYlor, J. L., \& MCCloskey, D. I. (1992). Ankle stiffness of standing humans in response to imperceptible perturbations: Reflex and task dependent components. Journal of Physiology, 454, 533-547.

Goodwin, G. M., McCloskey, D. I., \& Matthews, P. B. C. (1972). The contribution of muscle afferents to kinaesthesia shown by vibration-induced illusions of movement and by the effects of paralysing joint afferents. Brain, 95, 705-748.

HALL, L. A., \& MCClOSKEY, D. I. (1983). Detections of movements imposed on finger, elbow and shoulder joints. Journal of Physiology, $335,519-533$.

LACKNER, J. R. (1988). Some proprioceptive influences on the perceptual representation of body shape and orientation. Brain, 111, 281-297.
Lestienne, F. \& Gurfinkel, V. S. (1988). Postural control in weightlessness: A dual process underlying adaptation to an unusual environment. Trends in Neurosciences, 11, 359-363.

Martin, J. H., \& Jessell, T. M. (1991). Modality coding in the somatic sensory system. In E. R. Kandel, J. H. Schwartz, \& T. M. Jessell (Eds.), Principles in neuroscience (pp. 341-352). New York: Elsevier.

Massion, J. (1992). Movement, posture and equilibrium: Interaction and coordination. Progress in Neurobiology, 38, 35-56.

MELVILl-JoNES, G., \& YouNG, L. R. (1978). Subjective detection of vertical acceleration: A velocity dependent response? Otolaryngologica, $85,45-53$.

NELSON, J. G. (1968). Effects of water immersion and body position on perception of the gravitational vertical. Aerospace Medicine, 39, 806-811.

Paillakd, J. (1987). Cognitive versus sensorimotor encoding of spatial information. In P. Ellen \& C. Thinus-Blanc (Eds.), Cognitive processes and spatial orientation in animal and man: Vol. 2. Neurophysiology and developmental aspects (pp. 43-77). Dordrecht: Martinus Nijhoff.

PaILLARD, J. (1990). Motor neglect: Issues and prospects. Review of Neurology, 146, 600-611.

Paillard, J. (1991). Knowing where and knowing how to get there. In J. Paillard (Ed.), Brain and space (pp. 461-481). Oxford: Oxford University Press.

Paulus, W. M., Straube, A., \& Brandt, Th. (1984). Visual stabilization of posture. Physiological stimulus characteristics and clinical aspects. Brain, 107, 1143-1163.

Plat, C. (1984). Cellular bases for gravistatic reception by invertebrates and vertebrates. In L. Bolis, R. Keynes, \& S. Maddreil S. (Eds.), Comparative physiology of sensory systems (pp. 563-586). New York: Cambridge University Press.

Pozzo, T., Berthoz, A., \& LeForT, L. (1990). Head stabilization during various locomotor tasks in humans: I. Normal subjects. Experimental Brain Research, 106, 82-97.

Riccio, G. E., Martin, E. J., \& Stoffregen, T. A. (1992). The role of balance dynamics in the active perception of orientation. Journal of Experimental Psychology: Human Perception \& Performance, 18, 624644.

Roll, J. P., \& Roll, R. (1988). Extraocular proprioception and body postural references. In V. S. Gurfinkel, M. E. Loffé, J. Massion, \& J. P. Roll (Eds.), Stance and motion: Facts and concepts (pp. 23-26). New York: Plenum.

Ross, H. E., Crickmar, S. D., Sills, N. V., \& Owen, E. P. (1969). Orientation to the vertical in free divers. Aerospace Medicine, 95, 728-732.

STOFFrEGEN, T. A., \& Riccio, G. E. (1988). An ecological theory of orientation and the vestibular system. Psychological Review, 95, 3-14.

Worringham, C. J., Stelmach, G. E., \& Martin, Z. E. (1987). Limb segment inclination sense in proprioception. Experimental Brain Research, 66, 653-658.

YounG, L. R. (1984). Perception of the body in space: Mechanisms. In I. Smith (Ed.), Handbook of physiology: Vol. 3. The nervous system (pp. 1023-1066). New York: Academic Press.

\section{NOTE}

1. To stimulate the visual system only, the subject's body and head were kept stationary and the visual system was stimulated by moving a room surrounding the subject. To stimulate the ankle proprioceptive system only, the subject's body and head were held stationary and ankle movements were stimulated by balancing a load using the ankles only.

(Manuscript received October 3, 1996; revision accepted for publication March 18, 1998.) 